\title{
MS10-P01 | A NeW 3D REFLECTION DATA VIEWER BASED ON NGL
}

Oeffner, Robert (University of Cambridge, Cambridge, GBR); Echols, Nathaniel (Molecular Biophysics and Integrated Bioimaging, Berkeley, CA, USA); Sammito, Massimo (Department of Haematology, aCambridge Institute for Medical Research, Cambridge, AUT); McCoy, Airlie J (University of Cambridge, Cambridge, GBR); Hatti, Kaushik (Department of Haematology, Cambridge Institute for Medical Research, Cambridge, GBR); Stockwell, Duncan H (Department of Haematology, Cambridge Institute for Medical Research, Cambridge, GBR); Croll, Tristan I (Department of Haematology, Cambridge Institute for Medical Research, Cambridge, GBR); Read, Randy (Department of Haematology, Cambridge Institute for Medical Research, University of Cambridge, Cambridge, GBR)

The 3D-Reflection data viewer in Phenix [1] is based on the OpenGL library which is deprecated on MacOS. A replacement based on supported libraries, was urgently required. The NGL-HKL-viewer has been developed in a way that leverages the codebase of the original viewer while extending the functionality.

Protein crystallography involves processing raw X-ray images to indexed intensities. Subsequent steps may associate additional data with these indices. As part of the changes to the 3D-Reflection data viewer the types of reflection data that can be displayed were expanded.

Real valued data are displayed as spheres scaled according to their magnitudes. Colour coding through both hue and saturation can be applied as well. The viewer allows associating one data parameter with the sizes of the spheres but colour them according to another data parameter. For example, phased structure factors can be displayed as spheres scaled by amplitudes, coloured according to phases and colour saturation used to represent figures of merit. Data can be sorted into bins and be shown or hidden.

NGL-HKL-viewer is scriptable from Python and part of CCTBX [2]. It is based on NGL [3] and therefore portable to all computing platforms with a modern web browser. The viewer can be embedded in graphical user interfaces such as Qt5 or PySide. It is part of Phaser.Voyager [4].

[1] Echols et al. J. Appl. Cryst. (2012). 45, 581

[2] Grosse-Kunstleve et al. J. Appl. Cryst. (2002). 35, 126.

[3] Rose et al. Bioinformatics (2018). 34, 3755

[4] Read et al. per comms 